\title{
ESTRÉS OXIDATIVO Y PATOGENIA DEL DAÑO ARTICULAR EN EQUINOS
}

\author{
OXIDATIVE STRESS AND EQUINE JOINT DAMAGE PATHOGENESIS
}

\author{
ANDrea MÜLleR ${ }^{1}$, Marco Galleguillos ${ }^{1}$, MaXimiano LemaîTre ${ }^{1}$, \\ MARÍA EUGENIA LETELIER ${ }^{2}$, HÉCTOR AdARMES ${ }^{1}$
}

\begin{abstract}
This review describe the sinovial joint and the different structures that are present in the pathogenesis of joint disease, such as synovial membrane, articular cartilage, and synovial fluid. Oxidative stress is involved in the pathogenesis and leads to increased risk for osteoarthritis by damaging many component of the joint, so there are different antioxidants mechanism, enzymatic and not enzymatic, that inhibit joint damage. Hyaluronic acid, chondroitin-sulfate and glutathione-transferases are described as antioxidant molecules present in synovial fluid.
\end{abstract}

KEY WORDS: Joint, osteoarthritis, oxidative stress, antioxidants.

Palabras ClaVe: Articulación, osteoartritis, estrés oxidativo, antioxidantes.

\section{INTRODUCCIÓN}

La claudicación asociada al trauma articular constituye uno de los factores más importante de invalidez y pérdida de la capacidad de trabajo del equino, por lo que la comprensión de los mecanismos involucrados en el proceso degenerativo articular es vital para desarrollar estrategias de prevención y tratamiento (Goodrich y Nixon, 2006).

\section{Caracterización de la Articulación Metacarpo- falángica Equina}

Los miembros torácicos del equino soportan más del $70 \%$ del peso corporal, y por esto sus articulaciones sinoviales cursan habitualmente con procesos inflamatorios y degenerativos cuando se pierde la integridad de la articulación. Así, la articulación metacarpo-falángica es una de las articulaciones que sufre mayores alteraciones debido a su movilidad y a que debe soportar grandes fuerzas de compresión. La transmisión de fuerzas a través de esta articulación sinovial requiere de la integridad de las estructuras

1 Proyecto FIV /2007 No 12100101 4602018. Facultad de Ciencias Veterinarias y Pecuarias. Universidad de Chile. Fax 9785526, hadarmes@uchile.cl, casilla 2 correo 15, Santiago.

2 Facultad de Ciencias Químicas y Farmacéuticas. Universidad de Chile. que la componen, tales como ligamentos, tendones, membrana sinovial, cartílago articular y hueso subcondral (McIlwraith, 1996).

\section{Articulación Sinovial}

Una articulación sinovial está compuesta de varias estructuras que contribuyen al proceso degenerativo articular. La cápsula articular está formada por dos

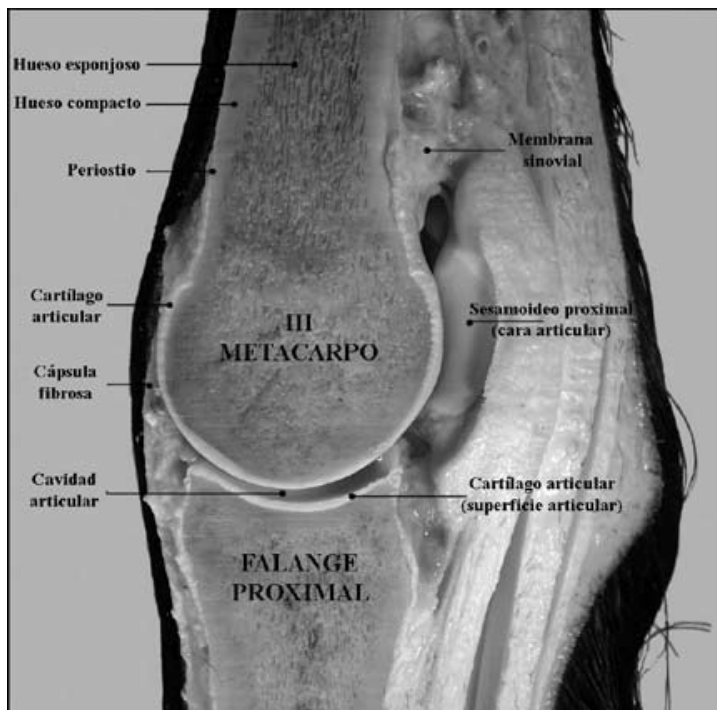

Figura 1: Fotografía corte sagital de la articulación metacarpofalángica equina (Maximiano Lemaître y Andrea Müller). 
capas, una externa compuesta de tejido fibroso y una interna, conocida como membrana sinovial. La capa fibrosa o membrana externa, está unida o muy próxima al borde de la cara articular (Getty, 1982).

La membrana sinovial reviste la cavidad articular excepto sobre los cartílagos articulares, ya que se inserta en el borde de estos cartílagos. Es una membrana delgada, muy irrigada e inervada, y frecuentemente forma pliegues y vellosidades que se proyectan dentro de la cavidad articular (Getty, 1982). Está formada por sinoviocitos de tipo A con actividad fagocítica y por sinoviocitos de tipo B que sintetizan el ácido hialurónico que forma parte del líquido sinovial (Pool, 1996). La membrana sinovial participa en la formación del líquido sinovial, que es un ultrafiltrado del plasma sanguíneo, por lo que su composición química es similar al plasma, al que se adiciona ácido hialurónico (Pool, 1996). Así, en el líquido sinovial se encuentran sinoviocitos y condrocitos, células derivadas de la membrana sinovial y del cartílago, respectivamente, y células propias del plasma sanguíneo, como monocitos, linfocitos y polimorfonucleares (PMN). Este líquido tiene múltiples funciones como son lubricar la articulación, transportar material nutricio hacia el cartílago articular, eliminar productos de desecho del cartílago y amortiguar las fuerzas de presión que soportan estas articulaciones. El líquido sinovial tiene la propiedad de ser muy viscoso debido a su contenido de ácido hialurónico, lo que otorga protección al cartílago al disminuir los efectos de fuerzas y roces, pero también reduce las interacciones entre enzimas, antígenos y citoquinas que pueden ejercer efectos dañinos sobre las células articulares cuando hay un proceso inflamatorio (Pool, 1996; Todhunter, 1996; Trotter y McIlwraith, 1996; Goodrich y Nixon, 2006).

Otro componente de las articulaciones es el cartílago articular de tipo hialino que recubre las superficies articulares óseas, disminuyendo el efecto de las contusiones violentas y del roce. El cartílago articular carece de nervios y de vasos sanguíneos, por lo que la nutrición de los condrocitos depende del líquido sinovial y de la irrigación del hueso subcondral, todo lo cual regula la función de estas células (Todhunter, 1996; Henrotin y col., 2005; Goodrich y Nixon, 2006; Milner $y$ col., 2006). El cartílago macroscópicamente posee una superficie lisa y un color azulado cuando se examina en fresco, cambiando estas características cuando hay procesos inflamatorios agudos y crónicos, apareciendo en este último caso focos de erosión y/o líneas de roce (Pool, 1996).

\section{Cartílago Articular}

El cartílago articular está compuesto de agua (75\%), colágeno $(15 \%)$, proteoglicanos $(10 \%)$ y condrocitos (2\%). El colágeno tipo II que predomina dentro del cartílago está constituido por tres cadenas alfa- 2 similares que forman finas fibrillas de 10 a 20 nanómetros de diámetro. Su función principal es la resistencia a la presión intermitente, que se asocia a las distintas fuerzas que actúan sobre las fibrillas de colágeno, ejerciendo tracciones y desplazamientos sobre ellas, provocando su compactación y su estiramiento (Ray y col., 1996).

Otro componente del cartílago son los proteoglicanos (PG) que están formados por una proteína central, a la que se unen covalentemente los glicosaminoglicanos (GAGs), siendo el agrecán el proteoglicano más abundante en el cartílago articular y el de mayor tamaño $\left(3 \times 10^{6} \mathrm{Da}\right)$. Los GAGs son polisacáridos heterogéneos formados por unidades repetitivas de al menos 2 monómeros distintos y cuyo carácter aniónico le permite atraer y retener agua en la matriz extracelular y le confieren, junto con el colágeno, sus propiedades viscoelásticas. Están representados por el ácido hialurónico, condroitín sulfato, keratán sulfato, heparán sulfato y dermatán sulfato I y II. El agrecán constituye el proteoglicano más importante cuantitativamente y se organiza formando estructuras supramoleculares de gran tamaño en la matriz extracelular (MEC) del cartílago articular, a través de su unión no covalente con una molécula de ácido hialurónico. Cada molécula de ácido hialurónico es capaz de unir hasta 100 moléculas de agrecán en forma no covalente. La formación de estos agregados es muy importante desde el punto de vista fisiológico, ya que permite la retención del agrecán y por ende de agua en la MEC del cartílago, asegurándose así la amortiguación normal del cartílago articular (Jackson et al., 1991; Todhunter, 1996).

\section{GAG'S presentes en la articulación}

El condroitín-sulfato es un GAG de gran tamaño producido por el condrocito, formado por unidades disacáridas de ácido glucurónico y N-acetil-D-galactosamina-sulfato. El condroitín sulfato es uno de los constituyentes principales del cartílago y se caracteriza por su extraordinaria capacidad de fijar agua. El condroitín-sulfato presente en el cartílago maduro es mayoritariamente condroitín-6-sulfato, a diferencia del condroitín-4-sulfato que se encuentra en mayor proporción en cartílagos inmaduros (Todhunter, 1996). En una articulación enferma, se observa 


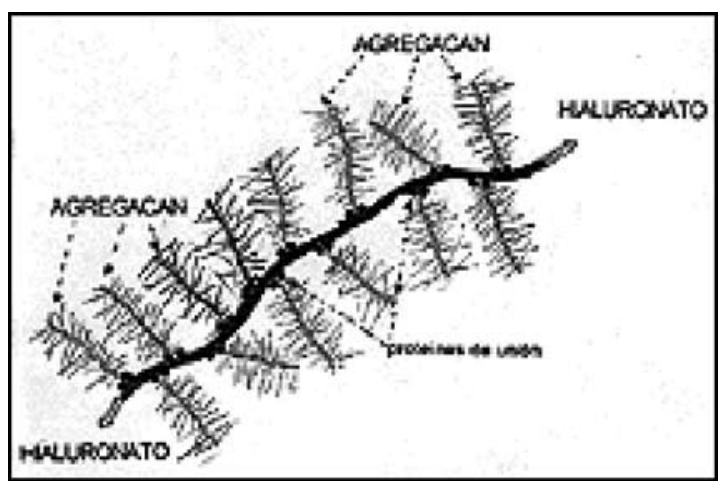

Figura 2: Esquema del Agrecan.

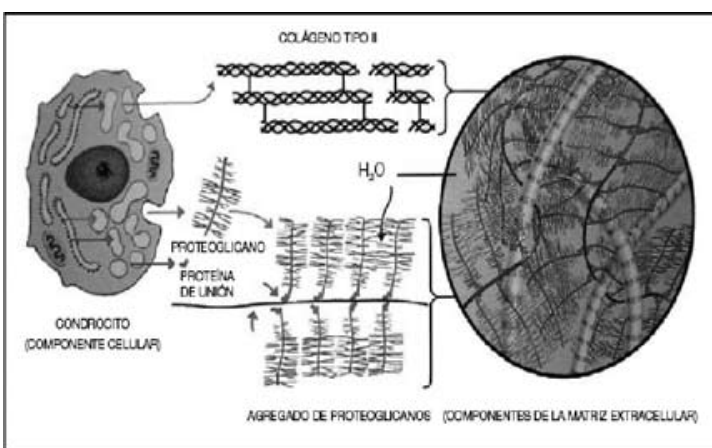

Figura 3: Esquema de la Matriz Extracelular.

pérdida de la estructura de la matriz extracelular (MEC) del cartílago, por lo que hay disminución de los componentes de ésta, como el condroitín-sulfato. En los procesos degenerativos, debido a la acción de enzimas líticas, se observa una degeneración progresiva del cartílago, produciendo como consecuencia una liberación de sus componentes, lo que genera pérdida de la capacidad para retener agua y un deterioro del funcionamiento articular.

Otro componente del cartílago articular es el ácido hialurónico, polímero de ácido glucurónico y $\mathrm{N}$-acetilglucosamina unidos alternativamente por enlaces glicosídicos (1-3) y (1-4). El ácido hialurónico presente en el cartílago es producido por los condrocitos, a diferencia de aquel presente en el líquido sinovial que es producido por los sinoviocitos (Howard y McIlwraith, 1996); difiere del resto de los GAGs debido a que carece de grupos sulfato. A través de su interacción con PG y con colágeno, proporciona estabilidad y elasticidad a la matriz extracelular del tejido conectivo. Además, se une por puentes de hidrógeno a diversas proteínas y moléculas de agua para formar un macroagregado viscoso que regula la homeostasis extracelular. El ácido hialurónico, por lo tanto, facilita el deslizamiento de las superficies articulares e impide el daño del cartílago articular
(Jackson y col., 1991; Balogh y col.; 2003; Campo y col., 2003a).

Todas las estructuras mencionadas de la articulación están involucradas en la enfermedad articular y sin importar la causa primaria todas ellas juegan un papel importante que contribuye a la patogénesis de la enfermedad articular (Goodrich y Nixon, 2006).

\section{Enfermedad Articular}

Un trauma, cualquiera sea su naturaleza, produce inflamación en el área afectada, que se traduce en aumento del flujo sanguíneo, aumento de la permeabilidad capilar y atracción de células fagocitarias al área dañada. La vasodilatación y el aumento del flujo sanguíneo originan el calor y el eritema en la zona. El aumento de la permeabilidad capilar lleva a una pérdida de fluido del sistema vascular hacia el tejido y el intersticio, generándose la tumoración característica en el área afectada. Este aumento de la permeabilidad facilita, además, la migración de células fagocitarias que liberan sus enzimas lisosomales al tejido dañado. Las células inflamatorias, como neutrófilos, macrófagos y linfocitos, a su vez, liberan mediadores proinflamatorios (citoquinas) que activan a otras células (Ostalowska y col., 2006).

Las citoquinas inflamatorias como IL-1 y TNF- $\alpha$ se liberan como respuesta al proceso inflamatorio, lo cual conduce a un aumento de la producción de proteinasas degradativas del cartílago articular y de prostaglandina $\mathrm{E}_{2}$; paralelamente, disminuye la concentración de proteoglicanos al aumentar su degradación y disminuir su síntesis. Estas consecuencias se deben a que la IL-1, en la matriz del cartílago articular, aumenta la concentración intracelular de fosfolipasa $\mathrm{A}_{2}$, que estimula la liberación de ácido araquidónico, sustrato de la ciclooxigenasa (COX2) que cataliza la síntesis de prostaglandina $\mathrm{E}_{2}\left(\mathrm{PG}-\mathrm{E}_{2}\right)$ que se libera al medio extracelular. El aumento de PG- $\mathrm{E}_{2}$ aumenta la concentración de $\mathrm{AMP}_{\mathrm{c}}$ intracelular, que estimula la producción de proteinasas (principalmente metaloproteinasas). En estas condiciones el condrocito disminuye la producción de colágeno tipo II y de proteoglicanos y aumenta la síntesis de enzimas degradativas llamadas metaloproteinasas (MMPs), principales mediadores en la degradación de la MEC. El proceso termina con una gran alteración de la MEC, lo que afecta finalmente la función articular (McIlwraith, 1996; Tsuji y col., 2006).

La siguiente figura esquematiza el proceso inflamatorio y las consecuencias en la articulación. 


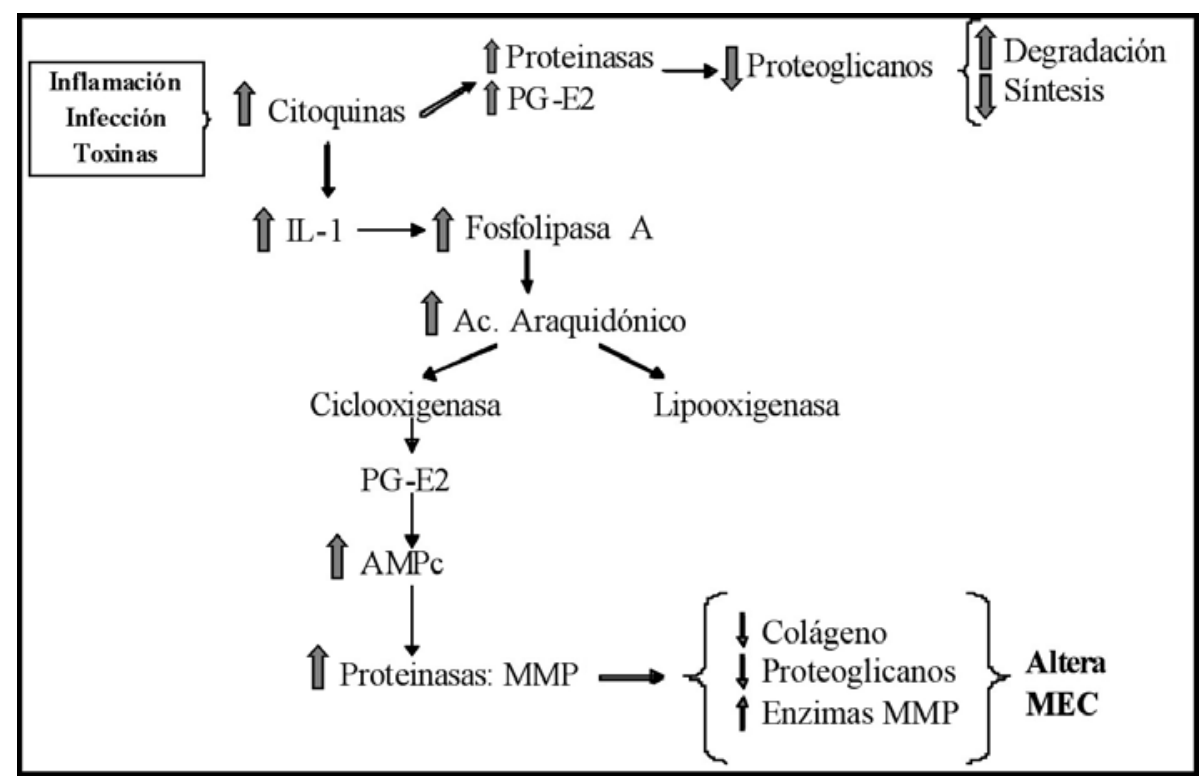

Figura 4: Cascada bioquímica del proceso inflamatorio.

La degeneración del cartílago se evidencia macroscópicamente por fibrilación, focos de erosión y líneas de roce sobre el cartílago articular (McIlwraith, 1996). Los cambios histológicos incluyen fibrilación superficial, que puede progresar para formar fisuras verticales hasta el hueso subcondral y necrosis de condrocitos (McIlwraith, 1996).

Los macrófagos, neutrófilos, linfocitos son los responsables de los procesos oxidativos conducentes a biodegradar las moléculas dañadas en el proceso inflamatorio. Ellos se activan mediante citoquinas inflamatorias, produciendo radicales libres que oxidan rápidamente las biomoléculas dañadas de la articulación (Campo y col., 2003a; Kirschvink y col., 2008). Este es un proceso fisiológico, que es primario a la reconstitución del tejido dañado y es regulado por la capacidad antioxidante celular. Sin embargo, cuando se prolonga en el tiempo, la capacidad antioxidante puede ser sobrepasada, condición en la cual se induce estrés oxidativo, resultando en un desbalance entre los agentes oxidantes y los antioxidantes (Sorg, 2004; Ostalowska, 2006).

\section{Papel de las Especies Reactivas al Oxígeno (EROS) en el daño articular}

Los EROS son especies oxidantes altamente reactivas $\mathrm{y}$, por tanto, capaces de degradar las biomoléculas, entre ellas los componentes de la articulación como son el colágeno, los proteoglicanos y el ácido hialurónico (Auer y col., 1993; Dimock y col., 2000; Ostalowska y col., 2006).
Las especies reactivas del oxígeno se generan por reducción del oxígeno molecular, lo cual puede originar especies radicalarias como el anión superóxido $\left(\mathrm{O}_{2}^{-}\right)$y el radical hidroxilo $(\mathrm{OH} \cdot)$. Estas son especies altamente reactivas y su vida media es muy corta. El $\mathrm{H}_{2} \mathrm{O}_{2}$, otra especie reactiva del oxígeno, no constituye un radical libre; ella se produce por dismutación del $\mathrm{O}_{2}$, reacción catalizada por la enzima superóxido dismutasa (SOD) (Lykkesfeldt y Svendsen, 2007; Valko y col., 2007).

Una de las vías a través de las cuales se forman EROS son las reacciones de Haber-Weiss y Fenton. En ellas los metales de transición como hierro o cobre en su estado iónico reaccionan con el oxígeno molecular generando las especies radicalarias del oxígeno (Sorg, 2004).

Ecuación de Haber-Weiss/Fenton:

$$
\begin{aligned}
& \mathrm{Fe}^{+3}+\text { ascorbato } \rightarrow \mathrm{Fe}^{+2}+\text { radical ascorbilo } \\
& \mathrm{Fe}^{+2}+\mathrm{O}_{2} \rightarrow \mathrm{Fe}^{+3}+\mathrm{O}_{2}^{--} \\
& \mathrm{Fe}^{+2}+\mathrm{H}_{2} \mathrm{O}_{2} \rightarrow \mathrm{Fe}^{+3}+\mathrm{HO}^{-}+\mathrm{HO}
\end{aligned}
$$

\section{Capacidad antioxidante celular}

La capacidad antioxidante celular está representada por mecanismos enzimáticos y no enzimáticos:

- Mecanismos enzimáticos. Las enzimas antioxidantes de mayor importancia son la catalasa (CAT), la superóxido dismutasa (SOD) y la glutatión peroxidasa (GPX).

- Mecanismos no enzimáticos. El Glutatión (GSH) y la vitamina E son los principales 
compuestos endógenos antioxidantes. Estos compuestos a diferencia de las enzimas, se consumen durante su acción antioxidante, por lo que deben ser regenerados después de ser oxidados.

En relación a las enzimas antioxidantes, la familia de las SOD está constituida por metaloenzimas que convierten el $\mathrm{O}_{2}{ }^{-}$en $\mathrm{H}_{2} \mathrm{O}_{2}$; las CAT transforman el $\mathrm{H}_{2} \mathrm{O}_{2}$ en agua y oxígeno molecular; y la GPX reduce hidroperóxidos a alcoholes y utiliza el GSH como agente reductor transformándolo en glutatión oxidado (GSSG). Cabe señalar que el GSSG es reducido nuevamente mediante la GSH-reductasa (GR) (Campo y col., 2003a; Sorg, 2004; Alfonso y col., 2007; Kirschvink y col., 2007; Lykkesfeldt y Svendsen, 2007). El siguiente esquema (Fig. 5) refleja la actividad de las diferentes enzimas con actividad antioxidante:

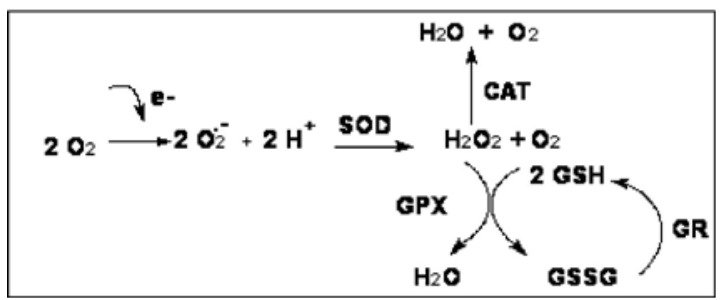

Figura 5: Actividad de enzimas antioxidantes.

Cabe señalar además que existe creciente bibliografía que señala a la GSH-transferasa (GSHt) como enzima reguladora de estrés oxidativo. Se describen isoformas de esta enzima, citosólica y ligadas a membrana. Todas están involucradas en la detoxificación de carcinógenos, contaminantes ambientales, agentes antitumorales e hidroperóxidos formados como metabolitos secundarios del estrés oxidativo que tienen como característica común, su alta electrofilicidad (Hayes y col., 2005). Estas proteínas catalizan además una variedad de reacciones de conjugación de compuestos electrofílicos y lipofílicos con glutatión (GSH). Más aún, las GSHt son capaces de unir reversiblemente una serie de compuestos lipofílicos y actuar como transportadores intracelulares, similar al rol que cumple la albúmina en la sangre; los compuestos que participan en esta unión reciben el nombre de ligandos. También estas proteínas unen covalentemente un amplio espectro de compuestos electrofílicos y lipofílicos en forma irreversible; posteriormente la proteína unida al electrófilo es degradada (Ketterey y Chritodoulides, 1969). Además, existen antecedentes que indican que la enzima microsómica (ligada a membrana del re- tículo endoplásmico hepático posee también actividad peroxidásica (Mosialou y Morgenstern, 1989)).

Todos los sistemas antioxidantes mencionados son fundamentales para mantener el equilibrio frente a un desbalance redox como el que ocurre en la enfermedad degenerativa articular. Existen diferentes estudios acerca de la capacidad antioxidante enzimática articular: superóxido dismutasa, catalasa, glutatión peroxidasa, glutatión reductasa, glutatiónS-transferasa (Alfonso y col., 2007; Ostalowska $y$ col., 2006).

Por otra parte, se ha propuesto que los glicosaminoglicanos, como el ácido hialurónico y el condroitin-4-sulfato podrían actuar como antioxidantes en la lesión articular (Balogh y col., 2003; Campo y col., 2003a; Campo y col., 2003b; Henrotin y col., 2003; Campo y col., 2004; Dechant y col., 2005). Sin embargo, se ha descrito que el contenido de ácido hialurónico tiende a disminuir en el líquido sinovial de articulaciones metacarpofalángicas severamente dañadas (Adarmes y col., 2006), pero otros autores han descrito un incremento de la capacidad antioxidante total (Dimock y col., 2000; Villasante, 2006).

\section{CONCLUSIONES}

Los antecedentes presentados muestran que no existe claridad sobre la capacidad antioxidante que posee la articulación. Es por ello que se podría evaluar la capacidad antioxidante del ácido hialurónico, el condroitín-sulfato y el líquido sinovial proveniente de articulaciones sanas y alteradas crónicamente, para conocer su real actividad antioxidante. La terapia antioxidante es eficaz en el tratamiento de patologías inflamatorias como la osteoartritis, pero debe ser combinada con tratamientos tradicionales. En el caso del equino, la suplementación con antioxidantes podría mejorar su estado general de salud, existiendo en el mercado suplementos orales que combinan ácido hialurónico, condroitín-sulfato, ácido arcórbico, entre otros.

\section{REFERENCIAS}

Adarmes, H.; Croxatto, A.; Gallegulllos, M.; GonZÁlez, E. 2006. Contenido de glicosaminoglicanos, aldehídos y proteínas en el líquido sinovial de la articulación metacarpofalángica equina normal y alterada. Arch. Med. Vet. 38(1): 47-52.

AuER, D.; NG, J. SEAWRIGHT, A. 1993. Free radical oxidation in plasma and synovial fluid of horses with synovial inflammation. Aust. Vet. J. 70: 49-52. 
Alfonso, V.; Champy, R.; Mitrovic, D.; Collin, P.; Lomri, A. 2007. Reactive oxygen species and superoxide dismutase: Role in joint disease. Joint Bone Spine. 74: 324-329.

BAlogh, G.; Illes, J.; Szekely, Z.; ForRai, E.; Gere, A. 2003. Effect of different metal ions on the oxidative damage and antioxidant capacity of hyaluronic acid. Arch. Biochem. Biophys. 410: 76-82.

CAmpo, G.; Avenoso, A.; CAmpo, S.; Ferlazzo, A.; Altavilla, D.; Micali, C.; CALATroni, A. 2003a. Aromatic trap analysis of free radicals production in experimental collagen-induced arthritis in the rat: protective effect of glycosaminoglycans treatment. Free. Radic. Res. 37(3): 257-268.

CAmpo, G. M.; Avenoso, A.; CAMPo, S.; FerlazZO, A. 2003b. Efficacy of treatment with glycosaminoglycans on experimental collagen-induced arthritis in rats. Arthritis Res. Ther. 5(3): 122-131.

Campo, G.; Avenoso, A.; Campo, S.; D’ascola, A.; Ferlazzo, A.; CAlatroni, A. 2004. The antioxidant and antifibrogenic effects of the glycosaminoglycans hyaluronic acid and chondroitin-4-sulphate in a subchronic rat model of carbon tetrachloride-induced liver fibrogenesis. Chem. Biol. Interact. 148: 125-138.

Dechant, J.E.; BAXTER, G.M.; FrISBIE, D.D.; TROTTER, G.W.; MCILWRAITH, C.W. 2005. Effects of glucosamine hydrochloride and chondroitin sulphate, alone and in combination, on normal and interleukin-1 conditioned equine articular cartilage explant metabolism. Equine Vet. J. 37: 227-231.

DiMOCK, A.N.; SiCILIANO, P.D.; MCILWRAITH, C.W. 2000. Evidence supporting an increase presence of reactive oxygen species in the diseased equine joint. Equine Vet. J. 32(5): 439-443.

GETTY, R. 1982. Sindesmología General (Artrología). En Anatomía de los Animales Domésticos. Sisson, S.; Grossman, J.D. Salvat

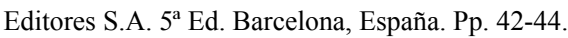

GOODRICH, L.R.; NIXON, A.J. 2006. Medical treatment of osteoarthritis in the horse -A review. Vet. J. 171: 51-69.

Hayes, J.D.; Flanagan, J.U.; Jowsey, I.R. 2005. Glutathione Transferases. Annu. Rev. Pharmacol. Toxicol. 45: 51-88.

Henrotin, Y.E.; Bruckner, P.; Pujol, J. P. L. 2003. The role of reactive oxygen species in homeostasis and degradation of cartilage. Osteoarthr. Cartil. 11(10): 747-755.

Henrotin, Y.; KurZ, B.; AigneR, T. 2005. Oxygen and reactive oxygen species in cartilage degradation: friends or foes? Osteoarthr. Cartil. 13: 643-654.

Howard, R.D.; MCILWRaITH C. 1996. Hyaluronan and its use in the treatment of equine joint disease. En McIlwraith, C.W., Trotter G.W. Joint disease in the horse. W.B. Saunders. Philadelphia, USA. Pp. 257-269.

JACKSON, R. L.; BUSCH, S. J.; CARDIN, A. D. 1991. Glycosaminoglycans: Molecular Properties, Protein Interactions, and role in Physiological Processes. Physiol. Rev. 71: 481-539.

Ketterery, B.; Chritodoulides, L. 1969. Two specific azodye carcinoge binding proteins of the rat liver: the identity of aminoacid residues which bind the azodye. Chem. Biol. Interact. 1:173-183.

KirschVInK, N.; De MoffarTs, B.; LeKeuX, P. 2008. The oxidant/ antioxidant equilibrium in horses. Vet. J. 177: 178-191.
Letelier, M.E.; Pimentel, A; Pino, P.; LePe, A.M.; FAúNDEZ, M.; ARACENA, P.; SPEISKY, H. 2005. Microsomal UDP-Glucuronyltransferase in Rat Liver: Oxidative Activation. Basic Clin. Pharmacol. Toxicol. 96: 480-486.

LYKKESFELDT, J.; SVENDSEN, O. 2007. Oxidants and antioxidants in disease: Oxidative stress in farm animals. Vet $\mathrm{J}$. 173: 502-511.

MCILWRAITH, C. 1996. General Pathobiology of the joint and response to injury. En McIlwraith, C.W., Trotter G.W. Joint disease in the horse. W.B. Saunders. Philadelphia, USA. Pp. 40-70.

Milner, P.I.; FAIRFAX, T.P.A.; BROWING, J.A.; WILKInS, R.J.; GIBSON, J.S. 2006. The effect of $\mathrm{O} 2$ tension on $\mathrm{pH}$ homeostasis in equine articular chondrocytes. Arthritis Rheum. 54: 3523-3532.

Mosialou, E.; Morgenstern, R. 1989. Activity of rat liver microsomal glutathione transferase toward products of lipid peroxidation and studies of the effect of inhibitors on glutathione-dependent protection against lipid peroxidation. Arch. Biochem. Biophys. 275 (1): 289-294.

Ostalowska, A.; Birkner, E.; Wiecha, M.; KASPERCZyK, S.; KASPERCZYK, A.; KAPOLKA, D.; ZON-GIEBEL, A. 2006. Lipid peroxidation and antioxidant enzymes in synovial fluid of patients with primary and secondary osteoarthritis of the knee joint. Osteoarthr. Cartil. 14: 139-145.

PooL, R.R. 1996. Pathologic manifestations of joint disease in the athletic horse. En McIlwraith, C.W., Trotter G.W. Joint disease in the horse. W.B. Saunders. Philadelphia, USA. Pp. 87-104.

RAY, C.S.; POOLE, A.R.; MCILWRAITH, C.W. 1996. Use of synovial fluid and serum markers in articular disease. En McIlwraith, C.W., Trotter G.W. Joint disease in the horse. W.B. Saunders. Philadelphia, USA. Pp. 203-216.

SORG, O. 2004. Oxidative stress: a theoretical model or a biological reality? C.R. Biol. 327: 649-662.

TODHUNTER, R.J. 1996. Anatomy and physiology of synovial joints. En McIlwraith, C.W., Trotter G.W. Joint disease in the horse. W.B. Saunders. Philadelphia, USA. Pp. 1-28.

Trotter, G.W.; MCIlWRAith, C.W. 1996. Clinical features and diagnosis of equine joint disease. En McIlwraith, C.W., Trotter G.W. Joint disease in the horse. W.B. Saunders. Philadelphia, USA. Pp. 120-145.

TSUJI, G.; KoSHIBA, M.; NAKAMURA, N.; KOSAKA, H.; HATACHI, S.; Kurimoto, C.; KurosaKa, M.; HaYAShi, Y.; YodoI, J.; KUMAGAI, S. 2006. Thioredoxin protects against joint destruction in a murine arthritis model. Free Radic. Biol. Med. 40: 1721-1731.

VAlKo, M.; LeIBFritZ, D.; MonCol, J.; Cronin, M.; MAZur, M.; TELSER, J. 2007. Free radicals and antioxidants in normal physiological functions and human disease. Int. J. Biochem. Cell Biol. 39: 44-84.

Villasante, A. 2006. Memoria de Título Médico Veterinario. Determinación de la capacidad antioxidante y del daño oxidativo en el líquido sinovial de la articulación metacarpofalángica equina macroscópicamente sana y con daño crónico, Santiago. Universidad de Chile. Facultad de Ciencias Veterinarias y Pecuarias. 